\title{
Detecting Bacterial Surface Organelles on Single Cells using Optical
}

\section{Tweezers}

Johan Zakrisson, ${ }^{\dagger}$ Bhupender Singh, ${ }^{+}$Pontus Svenmarker, ${ }^{\dagger}$ Krister Wiklund, ${ }^{\dagger}$ Hanqing Zhang, ${ }^{\dagger}$

Shoghik Hakobyan, ${ }^{\ddagger}$ Madeleine Ramstedt, ${ }^{\ddagger}$ and Magnus Andersson ${ }^{\dagger}, *$

${ }^{\dagger}$ Department of Physics, Umeå University, 90187 Umeå, Sweden

Department of Chemistry, Umeå University, 90187 Umeå, Sweden

*Corresponding author: Magnus Andersson, Department of Physics, Umeå University, Phone: +46 90786 6336, e-mail: magnus.andersson@umu.se

\section{Supplementary materials}
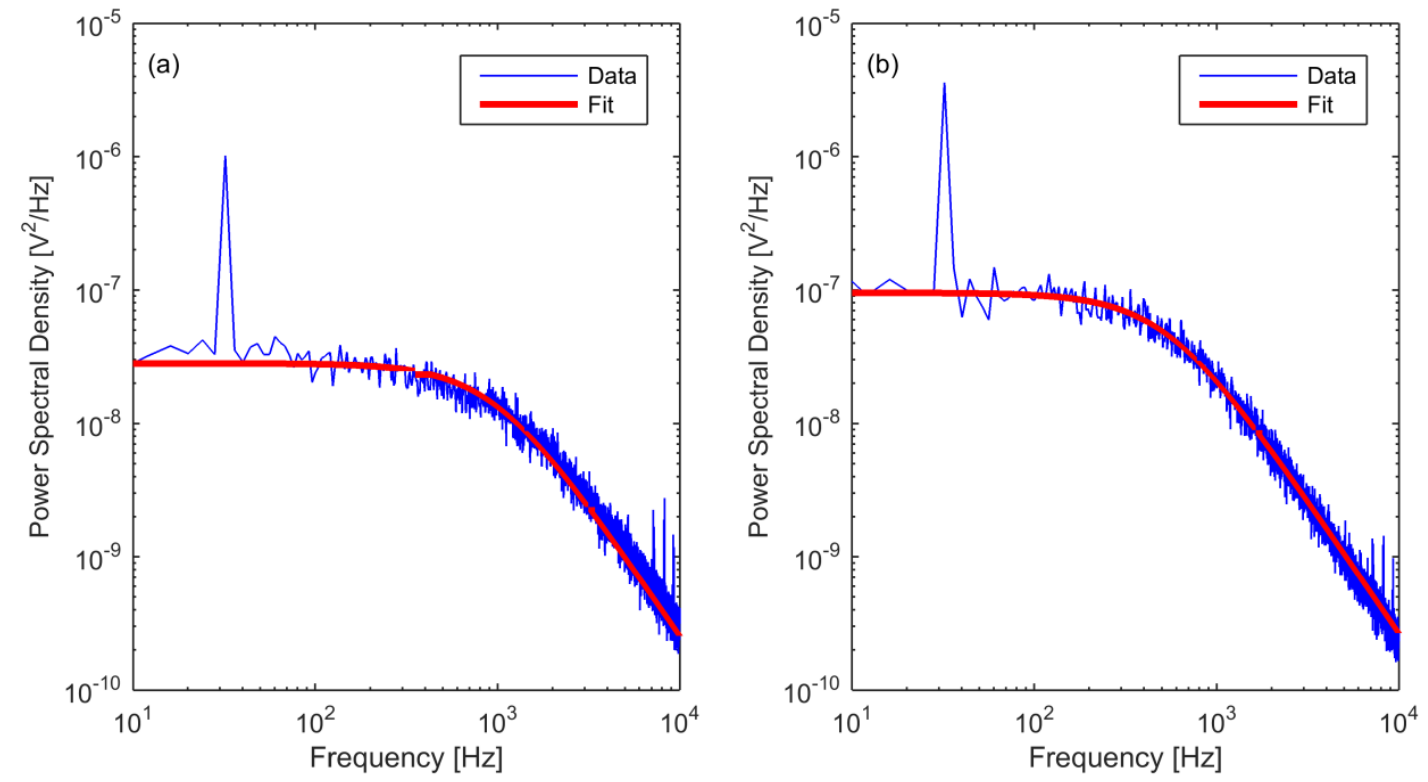

Figure S1. a) and b) show typical power spectra of trapped bacteria with the corresponding fits of Eq. 6 to the data. The fitted values from the power spectra are in a) $\beta$ equal to $1.24 \mu \mathrm{m} / \mathrm{V}$ and $D^{V}$ equal to 2.53 . $10^{-19} \mathrm{~V}^{2} / \mathrm{s}$ and in b) $\beta$ equal to $1.19 \mu \mathrm{m} / \mathrm{V}$ and $D^{V}$ equal to $2.63 \cdot 10^{-19} \mathrm{~V}^{2} / \mathrm{s}$. For both measurements, the temperature was $296 \mathrm{~K}$ and the viscosity was $0.932 \mathrm{mPa}$. 


\section{Quantification of bacterial shape}
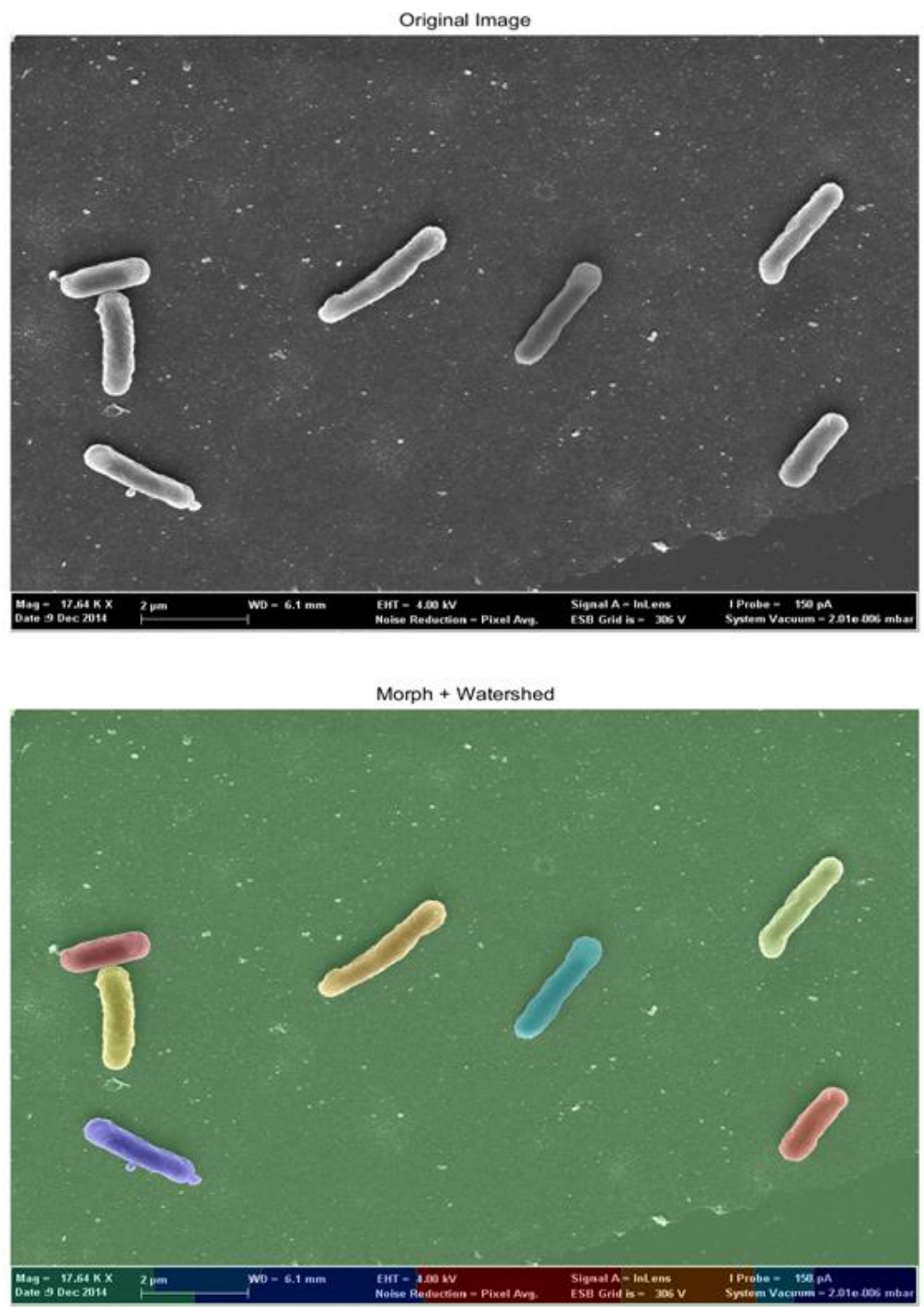

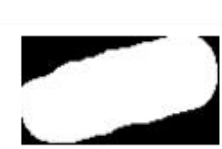

\#1

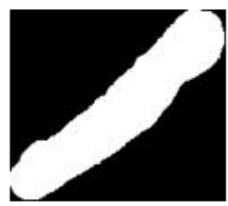

\#4

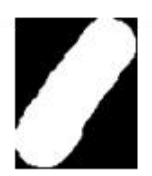

\#2

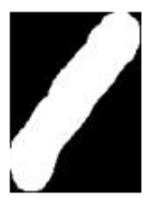

\#5

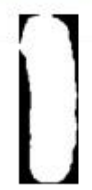

\#3

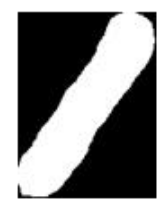

\#6

Figure S2. Example of bacteria segmentation. We used a standard segmentation algorithm to extract the contours of the bacteria. First, to extract the edge map from an original image (top), a Canny edge algorithm was used with two threshold values obtained by the Otsu's method. To create a dilated edge map, a morphological dilation operation using a $9 \times 9$ disk shaped kernel was used. Thereafter, a 
morphological reconstruction was conducted using the edge map from the Canny edge detection to find the boundaries, and the inversed dilated edge map to find the regional intensity minima. Finally, we applied the watershed algorithm to the reconstructed image, which results in a segmented image (middle) with segmented clusters marked in different colors. The edge maps of segmented bacteria were selected and further processed as shown in figure S3.

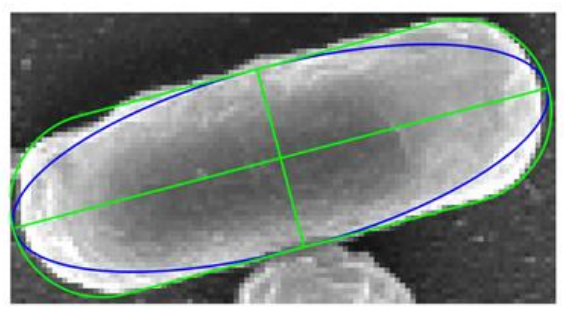

$\# 1$

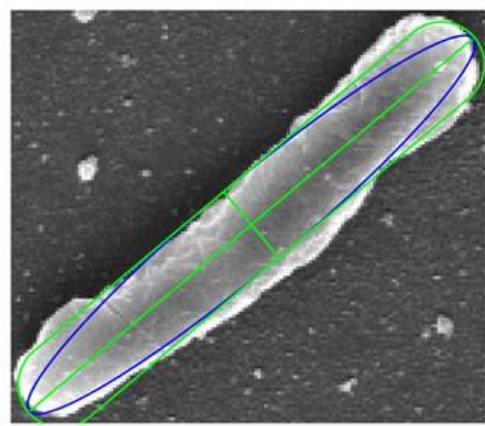

\#4

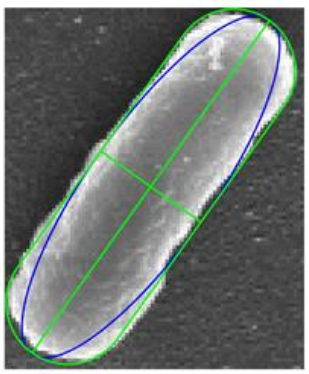

\#2

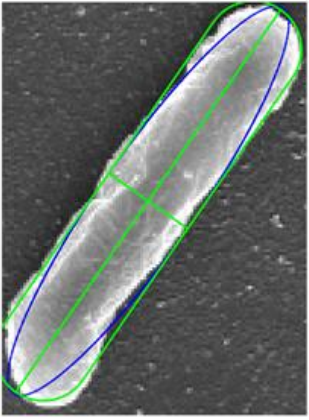

\#5

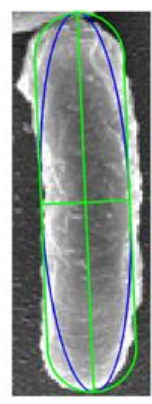

\#3

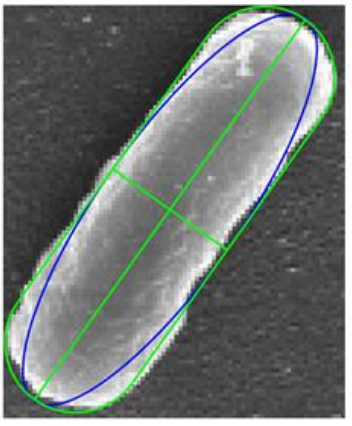

\#6

Figure S3. Demonstration of fitting of ellipses (blue curve) and projection of cylinders with spherical caps (green curve) based on the maximum and minimum axis length (green lines) of the segmented bacteria extracted from S2.

Table S1. Quantification of the fitting to the segmented area of the six bacteria in figure S3. We compared the binary template of segmented bacteria (shown in S2) and the area found by fitting the models in binary image form. We sum up all the pixels $\mathrm{N}_{\mathrm{d}}$ in the fitting model that differs from templates of the segmented bacteria, and divide it by the total number of pixels $N \times M$ in the template and thereby get $p=N_{d} /(N \times M)$. The result of the correctly fitted area in percentage is therefore 1-p.

\begin{tabular}{|c|c|c|c|c|c|c|}
\hline Fitting method Sample & $\mathbf{\# 1}$ & $\mathbf{\# 2}$ & $\mathbf{\# 3}$ & $\mathbf{\# 4}$ & $\mathbf{\# 5}$ & $\mathbf{\# 6}$ \\
\hline Ellipse & 86.19 & 87.98 & 77.61 & 89.12 & 91.41 & 89.57 \\
\hline $\begin{array}{c}\text { Projection of a cylinder } \\
\text { with spherical caps }\end{array}$ & 95.58 & 96.64 & 89.01 & 92.91 & 95.93 & 95.16 \\
\hline
\end{tabular}




\section{Microsphere size using brightfield microscopy}
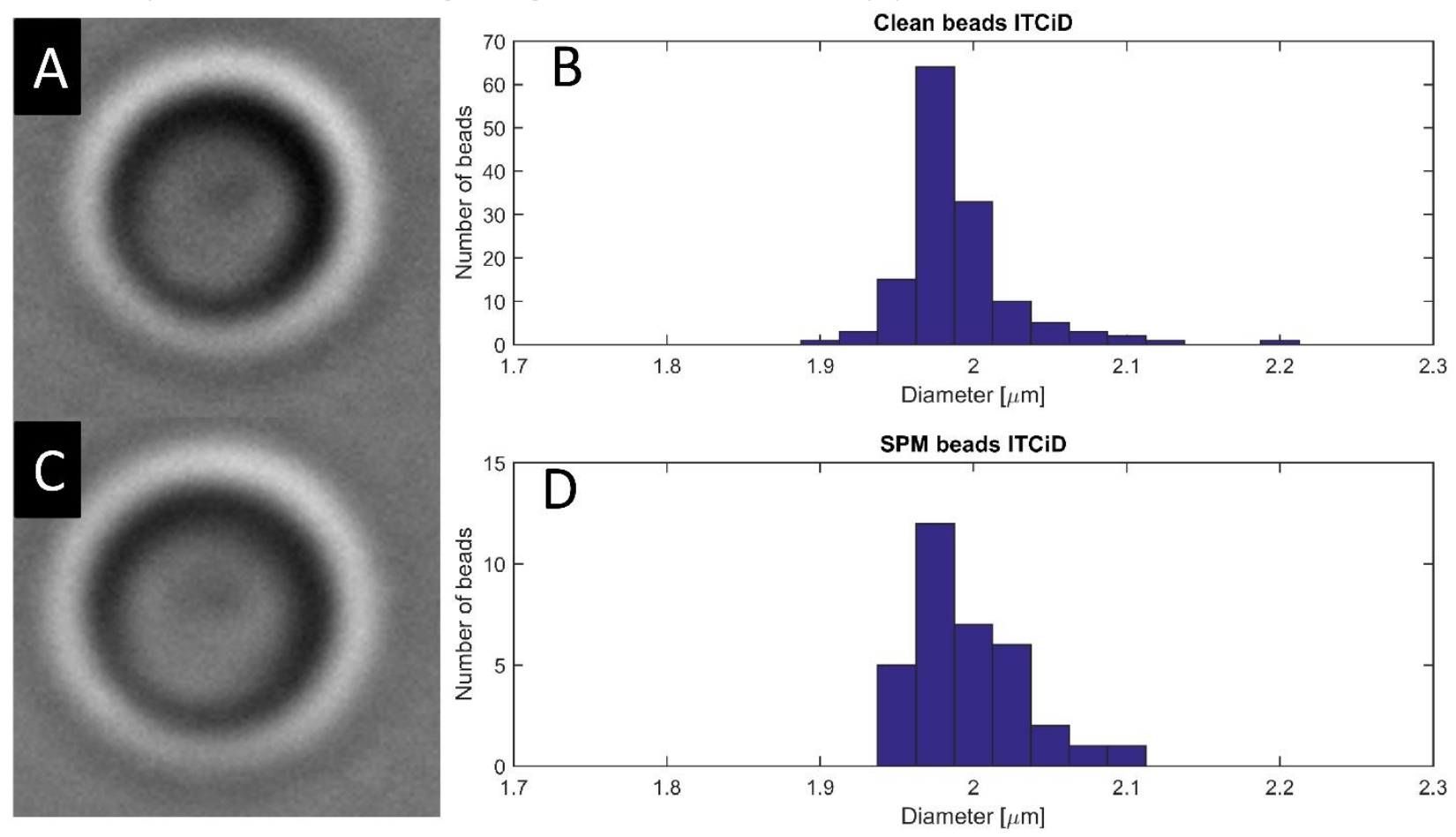

Figure S4. A) and C) show bright field microscopy images of pure (clean) and SPM coated microspheres. The histograms in B) and D) show the diameter of all investigated microspheres with average size of $1.989 \pm 0.003 \mu \mathrm{m}(\mathrm{n}=138$, mean $\pm \mathrm{SE}, \mathrm{SD}=0.04)$ and $1.997 \pm 0.007 \mu \mathrm{m}(\mathrm{n}=34$, mean $\pm \mathrm{SE}, \mathrm{SD}=0.04)$ for the pure and SPM coated microspheres, respectively. The diameter of a microsphere was assessed using the robust circle fitting algorithm, ITCiD. The ITCiD algorithm use a refinement stage that can improve the accuracy of finding the circle center and radius in comparison with the conventional Circular Hough transform. It is also capable of quantitating the 'completeness' of a circle by comparing the edge map with the estimated circle contour to check how well the projection of a microsphere fit to a circle. 


\section{Propagating error when quantitating the bacterial shape}

Bacterial cell shape was quantified using a segmentation and fitting procedure. The segmentation process contributes to the largest error, and the fitting error depends in turn on the segmentation. To estimate the segmentation error we used the same data set of clean microspheres $(n=115)$ from the robust ITCiD fit, using these as the ground truth, and compared that with the data from the segmentation process. Thus, we used the diameter from ITCiD and compared that with the equivalent diameter assessed from the segmentation using the Matlab function regionprop. This gave us a difference of the mean value of the diameter of $0.05 \mu \mathrm{m}$ for the $2.0 \mu \mathrm{m}$ microspheres, which corresponds to an error of $2.5 \%$.

The fitting error was estimated and based on the results from the function regionprop. The regionprop works as an alternative to ellipse fitting algorithm using least squares optimization. We used this function to extract information such as: orientation, major and minor axis for constructing the cylinders cap model and fitting the model to the image as shown in figure S3. Since the estimation of the fitting error is dependent on the segmentation results we estimated the upper level of the propagating error to $3.0 \%$.
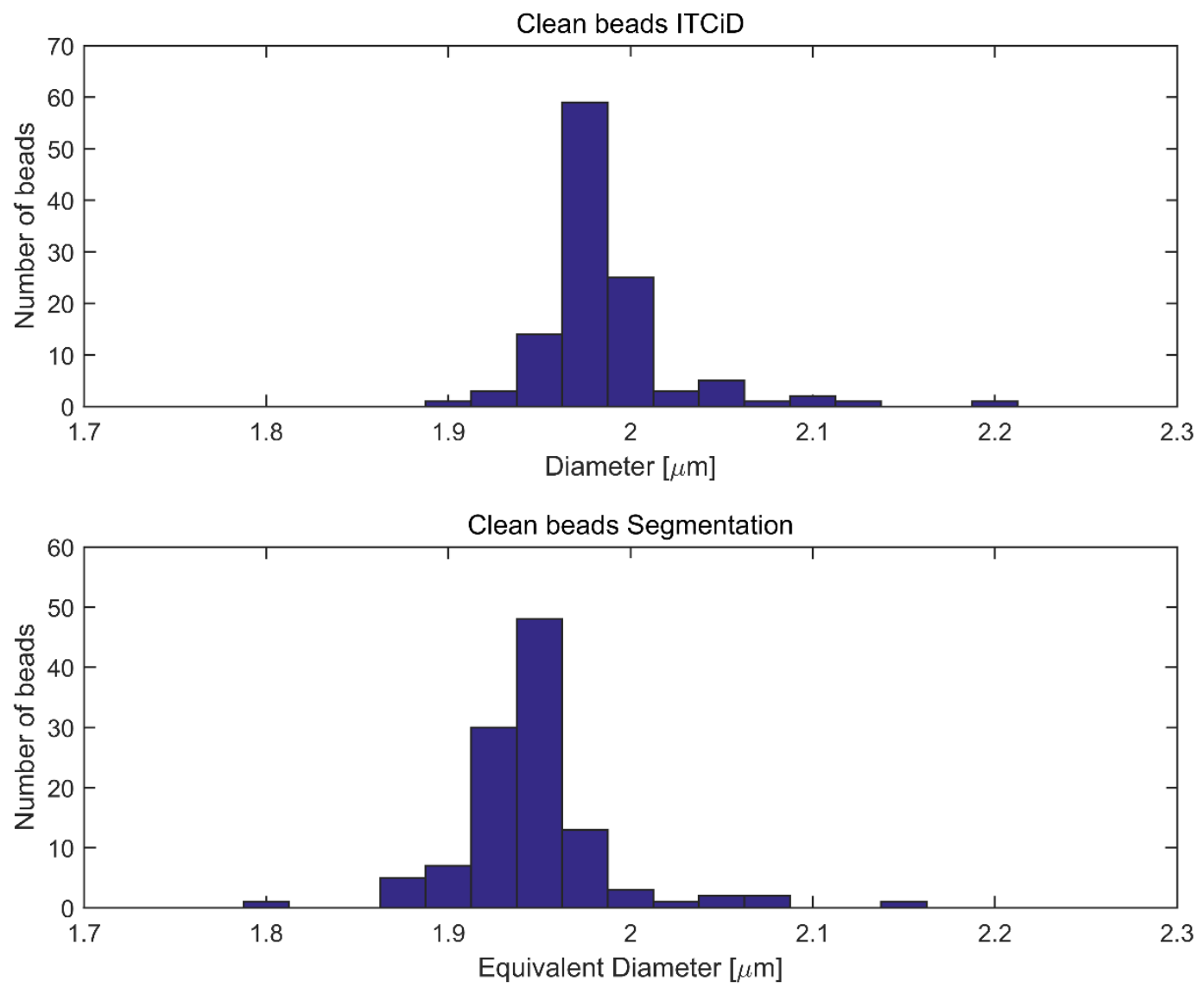

Figure S5. The histograms show the distribution of the estimated circle diameter using ITCiD (top) and segmentation (bottom). The mean of diameter is $1.985 \pm 0.004 \mu \mathrm{m}(\mathrm{n}=115$, mean $\pm \mathrm{SE}, \mathrm{SD}=0.0378)$ and $1.933 \pm 0.011 \mu \mathrm{m}(\mathrm{n}=115$, mean $\pm \mathrm{SE}, \mathrm{SD}=0.1203) \mu \mathrm{m}$ for ITCiD and segmentation method, respectively. 

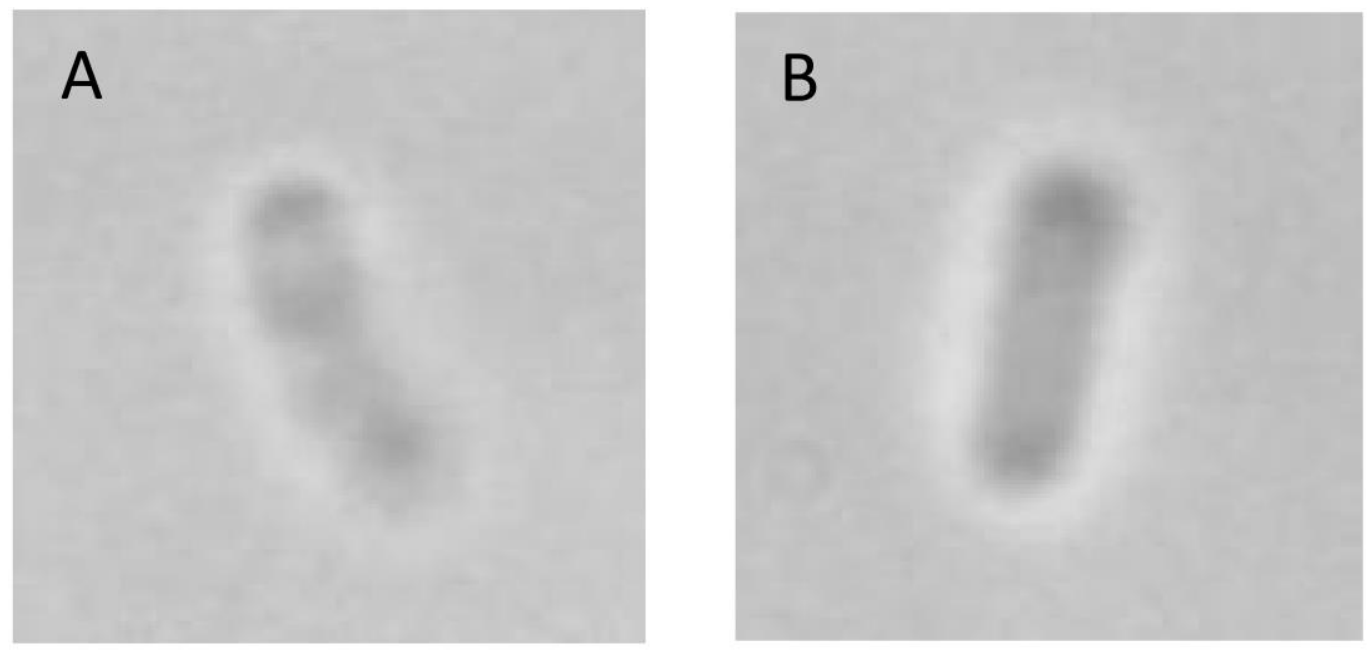

Figure S6. The left panel shows a bacterium formed as a "dumbbell", which did not provide a good fit between the experimental data and model. The right panel shows a typical bacterium, which model diameter aligns with the experimental diameter. 


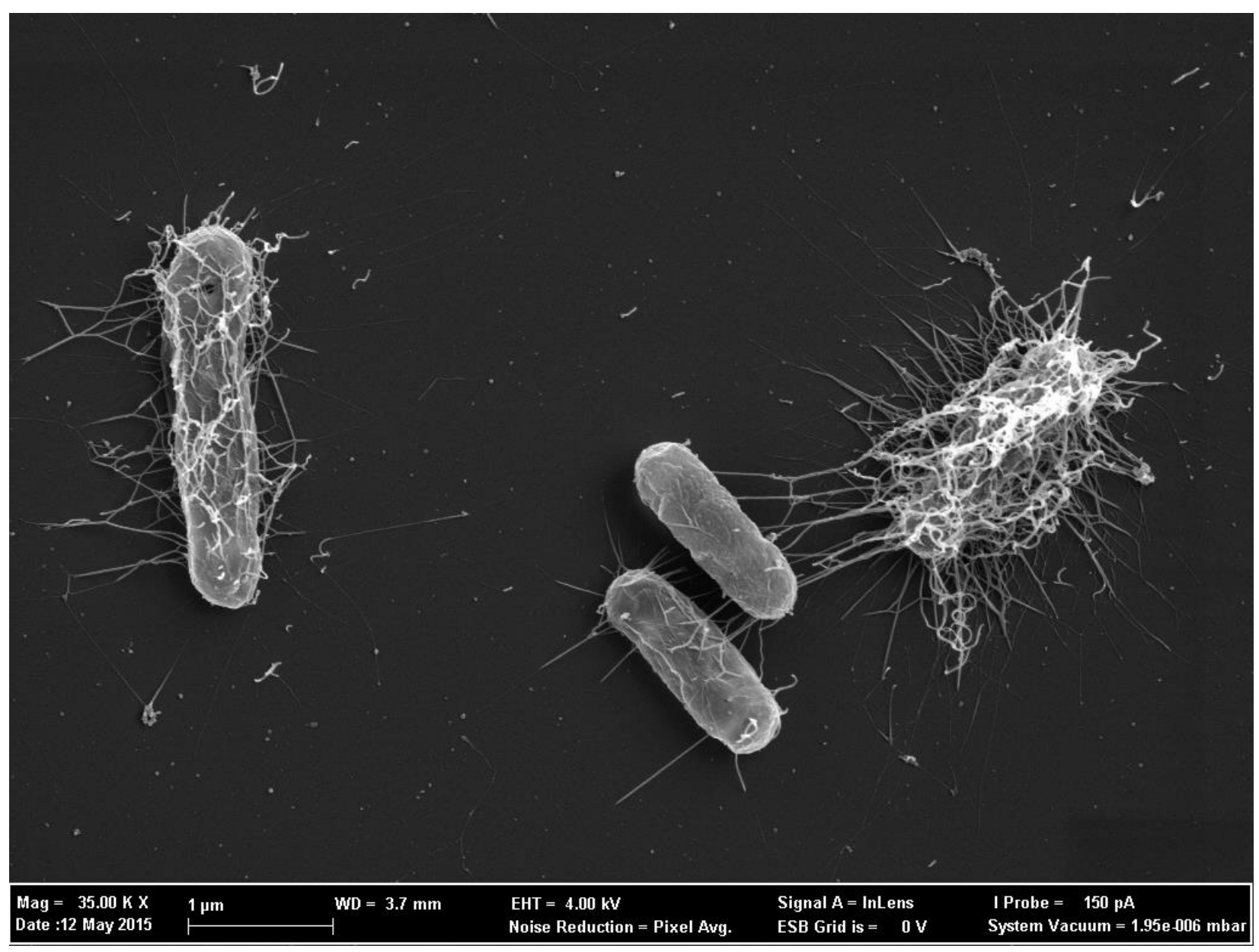

Figure S7. SEM micrograph of $S_{\text {II }}$ piliated bacteria. Note that the numbers of pili expressed by individual cells in a population vary, where some cells express very high (right), some express moderate (left) while some express a small amount of pili (middle). We suggest that the variation of the expression of pili in a cell population is related to the binomial distribution of kernel density plot in Figure 6B. 